\title{
DESAFIOS PARA ENFERMEIROS E FISIOTERAPEUTAS ASSISTIREM MULHERES IDOSAS COM INCONTINÊNCIA URINÁRIA
}

\author{
Andrelise Viana Rosa Tomasi ${ }^{1}$ \\ Silvia Maria Azevedo dos Santos ${ }^{1}$ \\ Gesilani Júlia da Silva Honório ${ }^{2}$ \\ Melissa Orlandi Honório Locks ${ }^{1}$
}

\author{
ORCID: https://orcid.org/0000-0003-3122-3365 \\ ORCID: https://orcid.org/0000-0001-9209-2894 \\ ORCID: https://orcid.org/0000-0001-5134-8697 \\ ORCID: https://orcid.org/0000-0003-0972-2053
}

Objetivo: conhecer como os enfermeiros e fisioterapeutas cuidam de mulheres idosas com incontinência urinária na Atenção Primária de Saúde. Método: estudo qualitativo, do tipo exploratório-descritivo, com análise temática descritiva. Foram realizadas entrevistas semiestruturadas com 10 enfermeiros e 14 fisioterapeutas atuantes em um Distrito Sanitário de Saúde de uma capital do Sul do Brasil. Resultados: os achados ratificam que o cuidado à população idosa fica fragmentado dentro da Atenção Primária de Saúde. Os idosos são atendidos de acordo com a doença crônica que possuem ou a queixa principal que apresentam. O mesmo ocorre com relação à incontinência urinária, que não é investigada nem priorizada no cuidado. Conclusão: é necessária uma educação permanente ou capacitação para que os profissionais possam responder aos desafios do envelhecimento populacional, em todos os aspectos da saúde, com vistas ao autocuidado.

Descritores: Incontinência Urinária; Enfermagem; Fisioterapia; Atenção Primária à Saúde.

\section{CHALLENGES FOR NURSES AND PHYSIOTHERAPEUTS TO ASSIST OLD WOMEN WITH URINARY INCONTINENCE}

Objective: To know how nurses and physiotherapists care for elderly women with urinary incontinence in Primary Health Care. Method: qualitative, exploratory-descriptive study, with descriptive thematic analysis. Semi-structured interviews were conducted with 10 nurses and 14 physiotherapists working in a Health District of a capital of southern Brazil. Results: the findings confirm that care for the elderly population is fragmented within Primary Health Care. The elderly are treated according to their chronic disease or their main complaint. The same is true of urinary incontinence, which is neither investigated nor prioritized in care. Conclusion: Continuing education or training is necessary for professionals to respond to the challenges of population aging, in all aspects of health, with a view to self-care.

Descriptors: Urinary Incontinence; Nursing; Physical Therapy Specialty; Primary Health Care.

\section{DESAFÍOS PARA LASENFERMERAS Y LOS FISIOTERAPEUAS PARAASISTIR A LAS MUJERES MAYORES COM INCONTINENCIA URINARIA}

Objectivo: conocer cómo las enfermeras y fisioterapeutas atienden a las mujeres mayores con incontinencia urinaria en atención primaria de salud. Metodo: estudio cualitativo, exploratorio-descriptivo, con análisis descriptivo temático. Se realizaron entrevistas semiestructuradas con 10 enfermeras y 14 fisioterapeutas que trabajan en un distrito de salud de una capital del sur de Brasil. Resultados: los hallazgos confirman que la atención a la población anciana está fragmentada dentro de la Atención Primaria de Salud. Los ancianos son tratados de acuerdo con su enfermedad crónica o su queja principal. Lo mismo ocurre con la incontinencia urinaria, que no se investiga ni se prioriza en la atención. Conclusión: la educación o capacitación continua es necesaria para que los profesionales respondan a los desafios del envejecimiento de la población, en todos los aspectos de la salud, con miras al autocuidado.

Descriptores: Incontinencia Urinaria; Enfermería; Fisioterapia; Atención Primaria de Salud.

Universidade Federal de Santa Catarina, SC

${ }^{2}$ Universidade Estadual de Santa Catarina, SC

Autor Correspondente Andrelise Viana Rosa Tomasi Email: andrelisev@gmail.com

Recebido: 10/09/2019

Aceito: 07/11/2019 


\section{INTRODUÇÃO}

O processo de transição demográfica e epidemiológica nos últimos anos no Brasil traz questões decisivas para os gestores, pesquisadores e profissionais da saúde. Contudo, ○ aumento de pessoas idosas é um fenômeno mundial, que ocorre de forma acelerada ${ }^{(1)}$. Devido a essa transição, sobreveio também uma alteração epidemiológica, ou seja, as doenças infectocontagiosas diminuiram e aumentaram as doenças crônicas não transmissiveis ${ }^{(2)}$.

Nesse contexto de transformações surgiram novos desafios para o cuidado. Os profissionais precisam estar preparados para atender as necessidades desse grupo populacional, uma vez que exigem cuidados que vão se tornando cada vez mais complexos com a idade.

Uma das condições que intervém na saúde do idoso são os problemas identificados na geriatria, como as sindromes independentemente de doenças específicas, como é o caso da incontinência urinária (IU), que afeta pessoas de qualquer idade, mas de forma mais enfática à população idosa. Esse problema atinge principalmente as mulheres idosas, uma vez que estão mais predispostas aos fatores de risco(3,4).

Diante do exposto, os cuidados para a IU são importantes para a assistência na APS, os sintomas causam desconfortos, perda da autoconfiança, interferindo de forma negativa na qualidade de vida. Ainda que, geralmente, ocorre uma subnotificação desse agravo, a presença do mesmo é muito frequente, de 30 a 60\%, dependendo do grau de fragilidade, podendo representar um problema de saúde pública $a^{(5)}$.

A sensibilidade dos profissionais da APS para investigar a ocorrência de IU é fundamental, nesse nível de atenção que podem ser concentrados os esforços no sentido de desenvolver ações buscando a prevenção da IU e/ou minimizando complicações e danos à saúde. Os profissionais da enfermagem e fisioterapia possuem um papel essencial, por terem a oportunidade de identificar e tratar o problema da IU, além de poderem trabalhar com estratégias para orientações e autocuidado(6.7).

Essas questões demonstram a necessidade de aprofundar os aspectos que circundam a assistência recebida pelas mulheres idosas com IU na APS. Frente a isso, este estudo teve como objetivo: conhecer como os enfermeiros e fisioterapeutas que cuidam de mulheres idosas com incontinência urinária na Atenção Primária de Saúde.

\section{METODOLOGIA}

\section{Tipo de estudo}

Trata-se de um estudo exploratório-descritivo com abordagem qualitativa.

\section{Participantes da pesquisa}

O estudo contou com uma população de 10 enfermeiros e 14 fisioterapeutas, respeitando os critérios de inclusão e disponibilidade dos participantes. Os critérios de inclusão para os enfermeiros: ser enfermeiro do Distrito Sanitário do Centro e estar desenvolvendo atividades assistenciais no mínimo há quatro meses. E para os fisioterapeutas: ser fisioterapeuta do Município, e estar desenvolvendo atividades assistenciais no mínimo há quatro meses.

\section{Local do estudo}

O local escolhido para realização da pesquisa foi um Distrito Sanitário de Saúde de uma capital do Sul do Brasil. Os dados foram coletados com enfermeiros e fisioterapeutas da Secretaria Municipal de Saúde.

\section{Coleta de dados}

A coleta de informações se deu em um Distrito Sanitário de Saúde de uma capital do Sul do Brasil, por meio de entrevista (semiestruturada) no período de agosto-outubro de 2014. A coleta de dados foi realizada no dia e horário previamente agendado nos centros de saúde, conforme a preferência do participante. Após a assinatura do Termo de Consentimento Livre e Esclarecido, as entrevistas foram gravadas em áudio e então, posteriormente, transcritas na integra pela pesquisadora responsável.

Os depoimentos foram identificados pelas letras "E" de enfermeiros e "F" de fisioterapeutas, seguidos por números sequenciais (El, E2 ... Fl, F2...), preservando o anonimato dos participantes.

\section{Procedimentos de análise dos dados}

O processo de análise de dados deu-se a partir da análise temática, sendo essa dividida em três etapas: pré-análise, exploração do material e tratamento dos resultados obtidos ${ }^{(8)}$. A análise das informações dos participantes emergiu a elaboração de dois eixos temáticos: A invisibilidade do idoso e a IU; e Desafios para assistir mulheres idosas com IU.

\section{Procedimentos éticos}

A pesquisa envolveu seres humanos, o que preconiza conformidade com a Resolução no 466/2012(9), sendo aprovado sob o parecer no 766.045 de 26 de agosto de 2014 . Os participantes receberam informações claras e concisas sobre a justificativa e objetivos referentes à pesquisa.

\section{RESULTADOS}

Dos 24 participantes entrevistados, dos quais 10 enfermeiros e 14 fisioterapeutas, 21 eram do sexo feminino. O tempo de trabalho na Prefeitura do Município variou entre quatro meses e dezessete anos.

Após o processo de análise dos dados, chegou-se a dois eixos temáticos, que cada um desses eixos é sustentado por um conjunto de categorias que buscam responder o objetivo. 


\section{A invisibilidade do idoso e da IU}

Durante esta pesquisa pôde-se observar que não há um olhar da pessoa idosa na sua integralidade por parte dos enfermeiros e fisioterapeutas, o que caracterizou uma fragmentação do cuidado ao idoso. Esse aspecto pôde ser observado nas emissões abaixo:

"A gente atende mulheres idosas, não tem assim um atendimento, vaga agendada para mulheres idosas, [...], como marcador de diabetes, foco mais voltado para atendimento nos grupos de prevenção de educação em saúde." (E3)

"A mulher idosa procura devido às dores musculares, queixas em relação à redução de mobilidade [...], coluna, membro superior e joelho, bastante demanda relacionados com problemas ortopédicos." (F13)

Por outro lado, ocorreu o relato de um participante que demonstrou que, quando tem algum profissional atento a saúde do idoso, outros aspectos são investigados e evidenciados nas consultas:

"Para essa questão da incontinência vai depender muito do Centro de Saúde, a gente tinha uma médica, que ela tem um interesse muito grande pela questão da incontinência, ela sensibilizou toda a equipe, aí veio essa demanda, pra eu fazer uma capacitação com a equipe." (F7)

Devido a isso, foi demonstrado algumas dificuldades nos atendimentos à pessoa idosa com IU, como podemos identificar na próxima categoria, que aborda as dificuldades identificadas pelos profissionais na assistência da mulher idosa com IU. Conforme se observa nas falas:

"Teria que ter uma estrutura mínima, aquisição de equipamentos, trazer profissionais com maior conhecimento ou especialistas [...]." (F13)

"Acho que a principal barreira é o não enxergar essa população." (F7)

Aqui foram relatadas as dificuldades pelos fisioterapeutas para atender mulheres idosas que possuem IU. Segundo os participantes, os problemas são: a falta de estrutura física adequada, a grande demanda de outros atendimentos e a falta de horário para agenda. Os profissionais não se sentem habilitados ou competentes para atender estas demandas.

Frente às dificuldades relatadas pelos profissionais, verificou-se que as estratégias utilizadas eram o encaminhamento para outros profissionais. O que vamos verificar na categoria seguinte é justamente como se davam a prática dos enfermeiros e fisioterapeutas sobre a referência e contra referência da assistência de mulheres idosas com IU.

A estratégia mais utilizada quando os pacientes com IU eram identificados na APS, era a de encaminhamento a serviços parceiros da Secretaria Municipal de Saúde e/ou aos médicos especialistas. Essa condição está relatada a seguir:

"Não, esse feedback a gente não tem, é bem dificil, a demanda é muito grande." (F8)

"Não tive retorno de nenhuma, outra que eu sei que fez cirurgia, mas a gente não tem registrado [...]para saber o que aconteceu depois, se foram na ginecologista ou fazer fisioterapia." (E2)

Assim, ficou evidente que os profissionais não tinham condições de prover esse atendimento, portanto realizavam o encaminhamento, mas não faziam o acompanhamento. Visto que o exercício prático de referência e contrarreferência se encontram pouco desenvolvido. Diante disso, surgiu outro eixo temático que aborda como esses profissionais realizam a assistência de mulheres idosas com IU.

\section{Como assistir mulheres idosas com IU}

Durante as entrevistas foi identificado que os enfermeiros e fisioterapeutas desenvolviam sua rotina de assistência no tocante às mulheres idosas com IU, o que emergiu a categoria o que fazemos. Alguns fragmentos das entrevistas apresentados a seguir:

"A gente orienta aqueles exercícios de abrir e fechar as pernas e fazer contração da vagina, urinar e soltar." (E6)

“Não faço atendimento porque a gente não tem essa área específica na prefeitura, são acolhidas, explico conforme os exercícios que podem fazer sozinhas em casa e são encaminhadas." (FI)

Fica evidenciado, de certa forma, que alguns profissionais compreendiam a ocorrência da IU, mas seria fundamental um conhecimento além dos exercícios de contração do períneo, ainda existem outros tratamentos conservadores e orientações que podem ser realizados. No entanto, essa condição é vista como uma especialidade, mas, se dentro da APS apresentarem conhecimentos consistentes em relação aos sintomas da IU, souberem identificar os diferentes tipos de IU e as possibilidades de tratamento, muitos casos poderiam ser minimizados.

Diante disso, a categoria a seguir discorre sobre o que os profissionais da APS identificaram como o que precisamos aprender em relação à IU. Em seus discursos, os informantes sugeriram a necessidade de uma capacitação, para qualificálos e melhorar a atenção à população idosa e, em especial, nos casos de IU. 
Portanto, não necessariamente os profissionais deverão se especializar em todas as áreas de atuação, mas, frente ao crescente aumento da população idosa, se faz necessário que todos busquem conhecimentos na área da gerontologia, pois, seguramente, irão assistir a idosos nos diferentes cenários de cuidado.

São múltiplas as possibilidades de tratamentos, levando em conta também as condições de saúde e a vontade de cura pelo paciente. Assim, segue abaixo a categoria sobre as estratégias para atender mulheres idosas com IU na APS.

A IU é uma realidade para muitas mulheres idosas e pode ter um impacto significativo no seu envelhecimento, interferindo diretamente na sua qualidade de vida. No entanto, a procura de cuidados para a IU ainda é pequena, e também não são investigadas, o que ficou evidenciado nas falas dos profissionais, conforme a seguir:

"Demanda sempre tem, de repente com a divulgação, porque às vezes elas acabam não falando muito, então acho interessante." (E4)

“Tem demanda, talvez agora não esteja aparecendo, mas existe, agora, por que será que os profissionais não tão conseguindo detectar ou porque não tem o que fazer $e$ deixa aquela demanda ali paradinho." (F9)

Esses relatos evidenciam que existe demanda, mas a mesma não é investigada e tratada de forma adequada. Frente a isso, pode-se perceber que as dificuldades em abordar as questões relativas à IU são tanto dos profissionais quanto dos pacientes que, em geral, têm vergonha de falar sobre o assunto. Portanto, entre as possibilidades futuras podemos destacar a necessidade de se estabelecer melhor o vínculo entre profissional e a pessoa idosa, para que esse assunto seja abordado de forma mais apropriada e tranquila dentro da APS.

Por outro lado, os profissionais deste estudo conseguiram identificar algumas facilidades para estratégias dentro da APS, para assistir mulheres idosas com IU, a partir do momento em que conseguiram identificar, abordar e direcionar um tratamento mais adequado para essa população, conforme as falas abaixo:

"Facilidades a gente percebe que muitas coisas simples podem ser resolvidas, desde que bem orientadas." (F8)

"Eu acho que é poder estar atuando com outros profissionais." (F9)

Percebe-se, assim, que os profissionais estavam cientes de que com uma capacitação na área gerontológica conseguiriam abordar melhor o tema da IU, além de implementarem condutas de cuidado com vistas à promoção da continência ou redução dos sintomas da IU.

\section{DISCUSSÃO}

Diante dos relatos do presente estudo, a população idosa é atendida como um marcador de doença, não sendo investigados outros problemas para se trabalhar com a promoção da saúde (PS), conforme estabelecido pela Política Nacional de Promoção da Saúde (PNPS), a qual se afirma que as ações públicas devem ir além da ideia da cura e reabilitação(10)

É através da PS que os profissionais têm condições de orientar as mulheres idosas, para assim proporcionar melhor qualidade de vida, nesse momento da senescência. Ainda nesse aspecto, pôde-se observar em relação ao cuidado de mulheres idosas com IU, aparece demanda na APS, mas a mesma ainda não é investigada rotineiramente. Este assunto poderia estar mais presente nas consultas de enfermagem e no próprio dia a dia dos demais profissionais da Estratégia de Saúde da Familia (ESF) e do Núcleo de Apoio à Saúde da Família (NASF), a prevalência de IU cada vez mais aumenta nessa população(5). Podemos, portanto, destacar que o fortalecimento das equipes de ESF é um ponto significativo para a atenção básica estruturada, com objetivo de fortalecer vínculos com os usuários, às famílias e a comunidade. Assim, poderão diminuir as fragilidades referentes à cobertura de ESF e dificuldades de estabelecer fluxos assistenciais bem definidos no acompanhamento(11)

Verifica-se também que quando uma equipe possui um profissional com olhar mais sensivel para as demandas gerontológicas, este pode motivar toda a equipe. No caso da IU, sugere-se uma abordagem multiprofissional, incluindo enfermeiros, médicos, fisioterapeutas e nutricionistas atuantes na APS, para favorecer a detecção precoce e o tratamento adequado desse agravo ${ }^{(12)}$.

Os índices de acometimento da IU em idosos são variados. Estudo transversal de base populacional, realizado com 1.593 idosos de um Município do Rio Grande do Sul, verificou a prevalência de IU de $20,7 \%$, sendo $26,9 \%$ entre as mulheres e $10,3 \%$ entre os homens ${ }^{(13)}$

Em outro estudo, realizado na Turquia, com objetivo de determinar a prevalência e os fatores de risco para a IU em 300 mulheres, 39\% dessas apresentavam IU, mas apenas 8\% tinham recebido algum tipo de tratamento(14). Em estudo na Região Norte da Califórnia, com 969 mulheres, verificaram que $65 \%$ dessas mulheres discutiam sobre sua IU com o clínico e eram as que tivessem clinicamente com os sintomas mais graves ${ }^{(15)}$.

Infelizmente, ainda existem casos que se encontram ocultos, negligenciados ou subtratados por parte dos profissionais da saúde, ocasionando efeitos negativos na qualidade de vida das pessoas afetadas, muitos profissionais consideram ser esse um problema de especialidade médica, não investigando sua ocorrência e nem buscando fazer algo pela população ${ }^{(16,17)}$. 
Outro estudo que investigou por quais motivos as mulheres não procuraram um tratamento para os sintomas da IU, verificou-se que grande parte das participantes não conhecia nenhum tipo de tratamento, as que conheciam só sabiam do procedimento cirúrgico(17).

Podemos perceber também que, apesar de se constituir como uma das bases de mudança no sistema de saúde, - exercício prático de referência e contrarreferência se encontrava pouco desenvolvido no presente estudo. A prática de encaminhamento com base nesse processo para as populações especificas, que visa o encaminhamento, ampliando-a para um processo de compartilhamento de casos e acompanhamento longitudinal de responsabilidade das equipes, atuando, portanto no fortalecimento de seus princípios(18). Visa à organização do fluxo de usuários e garantindo as ações de saúde. Pode ser considerada fundamental para a concretização do princípio da integralidade, mas essas técnicas assistenciais ainda são frágeis e isoladas ${ }^{(19)}$.

Portanto, quando não há a possibilidade dos encaminhamentos para outros profissionais especialistas, os participantes, dentro das diversas competências deveriam estar aptos para estabelecer um programa preventivo para IU(20)

Em um estudo, realizado com profissionais da saúde, incluindo médicos clínicos gerais, médicos da família e enfermeiros, constatou-se que esses raramente investigavam a presença de IU ou mesmo não o faziam. Além disso, quando ocorria alguma intervenção, a conduta era desconhecida ou não era a mais adequada(11). Este fato também foi discutido em revisão de literatura sobre os cuidados de mulheres idosas que apresentaram IU, verificouse que as mulheres mais velhas, quando apresentavam uma intensidade mínima dos sintomas urinários, não iam à procura de cuidados. Elas entendiam que essa condição fazia parte do processo de envelhecimento(21).

Por outro lado, na APS é possivel uma abordagem em equipe multidisciplinar onde os profissionais podem propor soluções em conjunto e assistir de maneira ampla toda a gama de cuidados possíveis para os sintomas da IU(22)

Os profissionais não se sentem habilitados ou com competência para atender estas demandas, é preciso ir à busca dessas capacitações. Em um estudo realizado com as práticas desenvolvidas por enfermeiros no âmbito da atenção básica, foram às atividades educativas que quando identificadas perpassam a PS, prevenção e controle de agravos, o autocuidado, e ainda orientações técnicas sobre a realização de procedimentos ${ }^{(23)}$

A PS, portanto, pode ser realizado por toda a equipe dentro da APS, desde que esses tenham conhecimentos acerca dasorientaçõescorretasquedevem serimplementadas caso a caso. Estudo realizado com 1475 mulheres idosas foi demonstrado que mudanças na composição corporal podem ser associadas com mudanças da predominância de IU. Esses achados sugerem que a otimização da composição corporal pode ser protetora contra a incidência, persistência e piora de IU. Essa informação é importante no aconselhamento dos fatores de risco para a IU(24)

Em relação a qualidade de vida e a IU, 22 mulheres idosas coreanas afirmaram que a IU gera perda de dignidade e problema de odor, e consideraram uma doença incontrolável e um comprometimento na vida. Essas mulheres usavam estratégias para gerenciar a IU, mas implementaram, principalmente, ações para manter a IU em segredo. Portanto, concluíram-se que abordagens educacionais são necessárias para informar às mulheres com IU sobre habilidades de gerenciamento mais eficazes ${ }^{(25)}$.

\section{Limitações do estudo}

A limitação desse estudo foi o pequeno número de enfermeiros e fisioterapeutas que participaram, e o não conhecimento desses profissionais em relação ao número de mulheres idosas com IU. Esperamos ter contribuído com o processo de reflexão e aprendizagem destes profissionais, de tal forma que consigam desenvolver uma assistência às mulheres com IU.

\section{Contribuição do estudo para a prática}

Acontribuição desta pesquisa é de extrema relevância para fomentar mais publicações relacionadas à IU em mulheres idosas na APS, principalmente no âmbito interdisciplinar.

\section{CONCLUSÃO}

Este estudo vem agregar novo conhecimento em relação à temática estudada, ao mostrar como os profissionais enfermeiros e fisioterapeutas assistem às mulheres idosas com IU, demonstrando a dificuldade em identificar, tratar orientar ou encaminhar essa população. Portanto, os resultados desta pesquisa revelam a necessidade desses profissionais aprimorarem seus conhecimentos, com vistas à melhoria no cuidado, de forma integral e contínua.

Verificou-se que existe necessidade de educação permanente ou uma capacitação para que os profissionais possam lidar com os desafios do envelhecimento. Para que não apenas saibam como assistir essa população, como também estejam preparados para prestar cuidado à mulher idosa com IU.

Contribuição dos autores: Concepção e desenho, análise e interpretação dos dados, redação do artigo: Andrelise Viana Rosa Tomasi, Silvia Maria Azevedo dos Santos; revisão crítica e revisão final: Silvia Maria Azevedo dos Santos, Gesilani Júlia da Silva Honório, Melissa Orlandi Honório Locks. 


\section{REFERÊNCIAS}

1. Veras R. Envelhecimento populacional contemporâneo: demandas, desafios e inovações. Rev.Saúde Pública. [Internet]. 2009 [citado 2018 Mar 15]:43(3):548-554. Disponivel em: http://www.scielo.br/pdf/rsp/ v43n3/224.pdf

2.Nasri F. O envelhecimento populacional no Brasil. Einstein. [Internet]. 2008 [citado 2018 Mar 15]; 2008; 6 (Supl1):S4-S6.Disponivel em: http:// apps.einstein.br/revista/arquivos/PDF/833-Einstein\%20Suplemento\%20v6nl\%20pS4-6.pdf

3.Moraes EN, Marino MCA, Santos RR. Principais sindromes geriátricas. Rev. Med. Minas Gerais [internet]. 2010 [citado 2018 Mar 17]:20(1):5466. Disponivel em: http://www.observatorionacionaldoidoso.fiocruz. br/biblioteca/_artigos/196.pdf

4.Pitangui ACR, Silva RG, Araújo RC. Prevalência e impacto da incontinência urinária na qualidade de vida de idosas institucionalizadas. Rev. Bras.Geriatr.Gerontol. [Internet]. 2012 [citado $2018 \mathrm{Mar}$ 19]: 15(4):619-626. Disponivel em: http://www.scielo.br/scielo.php?pi$d=\$ 1809-82320120004000028$ script=sci_abstract\&tlng=pt

5. Moraes EN. Atenção à saúde do idoso: aspectos conceituais. Brasilia: Organização Pan-Americana de Saúde. [Internet]. 2012 [citado 2018 Mar 15]. Disponivel em: https://apsredes.org/pdf/Saude-do-Idoso-WEBl.pdf

6. Langoni CS, KnorstMR, Lovatel GA, Leite VO, Resende TL. Incontinência urinária em idosas de Porto Alegre: sua prevalência e sua relação com a função muscular do assoalho pélvico. Fisioter. Pesq. [Internet]. 2014 [citado 2018 Mar 16]; 21(1):74-80. Disponivel em: http://www. scielo.br/scielo.php?pid=S1809-29502014000100074\&script=sci_arttext\&tlng=pt

7. Mata LRF, Gomes CRG, Goulart LC, Macedo MML, Rodrigues RN. National scientific production in nursing journals related to urinary incontinence: integrative review. Rev. Enferm. UFPE. [Internet]. 2014 [citado 2018 Mar 16]; 8(7):3188-3196. Disponivel em: https://periodicos.ufpe. $\mathrm{br} /$ revistas/revistaenfermagem/article/view/10042

8. Minayo MCS. O desafio do conhecimento: pesquisa qualitativa em saúde. 13.ed. São Paulo: Hucitec; 2013

9. Ministério da Saúde (BR). Diretrizes e normas regulamentadoras da pesquisa em seres humanos: Resolução 466/12 do Conselho Nacional de Saúde. Rio de Janeiro: FIOCRUZ. [Internet]. 2012 [citado 2017 Nov 14]. Disponivel em: https://bvsms.saude.gov.br/bvs/saudelegis/ cns/2013/res0466_12_12_2012.html

10. Ministério da Saúde (BR). Secretaria de Vigilância em Saúde. Secretaria de Atenção à Saúde. Política Nacional de Promoção da Saúde/ Ministério da Saúde, Secretaria de Vigilância em Saúde, Secretaria de Atenção à Saúde. 3. ed. Brasilia [Internet]. 2010 [citado 2018 Abr 05]. Disponivel em: http://bvsms.saude.gov.br/bvs/publicacoes/politica nacional_promocao_saude_3ed.pdf

11. Bandeira FJS, Campos ACV, Gonçalves LHT. Rede de atenção: fragilidades no processo de implementação na perspectiva de especialistas em gestão da atenção primária. Enferm.Foco. [internet]. 2019 [citado 2019 Set 04]; 10(2):24-29. Disponivel em: http://revista.cofen.gov.br/ index.php/enfermagem/article/view/1988/514

12. Barbosa SS, Oliveira LDR, Lima JLDA, Carvalho GM, Lopes MHBM. Como profissionais de saúde da rede básica identificam e tratam a incontinência urinária feminina. O Mundo da Saúde. [Internet]. 2009 [citado 2018 Abr 18]; 33(4):449-456. Disponivel em: http://www.saocamilo-sp.br/pdf/mundo_saude/70/449a456.pdf

13.KesslerM, Facchini LA, Soares MU, Nunes BP, França SM, Thumé $E$. Prevalência de incontinência urinária em idosos e relação com indicadores de saúde fisica e mental. Rev. Bras. Geriatr. Gerontol.[Internet]. 2018 [citado 2019 Jan 10]; 21(4):409-419. Disponivel em: http://www.scielo.br/scielo.php?script=sci_arttextEpi$d=$ S1809-98232018000400397\&lng=ptEnrm=iso\&tlng=pt
14. Gözükara F, Koruk İ, Kara B. Urinary incontinence among women registered with a family health center in the Southeastern Anatolia Region and the factors affecting its prevalence. Turk. J. Med. Sci. [Internet]. 2015 [citado 2018 Mar 20]:45(4):931-9. Disponivelem:http:// journals.tubitak.gov.tr/medical/issues/sag-15-45-4/sag-45-4-311410-31.pdf

15. Duralde ER, Walter LC, Van Den Eeden SK, Nakagawa S, Subak LL. Brown JS, et al. Bridging the Gap: Determinants of Undiagnosed or Untreated Urinary Incontinence in Women. Am. J. Obstet. Gynecol. [Internet]. 2015 [citado 2018 Mar 20]:pii:S0002-9378 (15) 01020-0. Disponivel em: http://ac.els-cdn. $\mathrm{com} / \mathrm{S} 0002937815010200 / 1-\mathrm{s} 2.0-$ S0002937815010200-main. pdf?_tid $=8 \mathrm{c} 39196 \mathrm{e}=8703-11-5 \mathrm{e}-744 \mathrm{a} 00000-\mathrm{aac} 362 \mathrm{~b} \varepsilon \mathrm{acdnat}-$ 1447088681_08badbd6blf6lc28efb5592cf6c9185a

16. Loureiro LSN, Medeiros ACT, Fernandes MGM, Nóbrega, MML. Incontinência urinária em mulheres idosas: determinantes, consequências e diagnósticos de enfermagem. Rev. Rene.[Internet]. 2011 [citado 2018 Mar 21];12(2):417-423. Disponivel em: http://www.redalyc.org/ articulo.oa?id=324027975019

17. Silva L. Lopes MHBM. Incontinência urinária em mulheres: razões da não procura por tratamento. Rev. Esc. Enferm. USP. [Internet]. 2009 [citado 2018 Abr 19]; 43(1):72-78.

Disponivel em:http://www.scielo.br/scielo.php?script=sci_arttextEpi$\mathrm{d}=$ S0080-62342009000100009

18. Ministério da Saúde (BR). Secretaria de Atenção à Saúde. Departamento de Atenção Básica. Política Nacional de Atenção Básica/Ministério da Saúde. Secretaria de Atenção à Saúde. Departamento de Atenção Básica. Brasilia. [Internet]. 2012 [citado 2018 Abr 05]. Disponivel em: http://189.28.128.100/dab/docs/publicacoes/geral/pnab.pdf 19. Fratini JRG, Saupe R, Massaroli A. Referência e contra referência: contribuição para a integralidade em saúde. Cienc. Cuid. Saúde. [Internet]. 2008 [citado 2018 Abr 21]; 7(1):65-72. Disponivel em: http:// 4908-Texto\%20do\%20artigo-14551-1-10-20080908\%20(1).pdf

20. Caldas CP, Conceição IRS, José RMC, Silva BMC. Terapia comportamental para incontinência urinária da mulher idosa: uma ação do enfermeiro. Texto Contexto Enferm. [Internet]. 2010 [citado 2018 Abr 21]; 19(4):783-788. Disponivel em: http://www.scielo.br/scielo. php?script=sci_arttextEpid=S0104-07072010000400023

21. Strickland, R. Reasons for not seeking care for urinary incontinence in older community-dwelling women. Urol. Nurs. [Internet]. 2014[citado 2018 Abr 21]; 34(2):63-68. Disponivel em: https://www. ncbi.nlm.nih.gov/pubmed/24919243

22. Oliveira EM, Spiri WC. Programa saúde da familia: a experiência de equipe multiprofissional. Rev.Saúde Pública. [Internet]. 2006 [citado 2018 Abr 21]:40(4):727-733.

Disponivel em:http://www.scielo.br/pdf/rsp/v40n4/25.pdf

23. Acioli S, KebianLVA, Faria MGA, Ferraccioli P, Correa VAF. Práticas de cuidado: o papel do enfermeiro na atenção básica. Rev. Enferm. UERJ. [Internet]. 2014 [citado 2018 Abr 21]; 22(5):637-642. Disponivel em: http://www.facenf.uerj.br/v22n5/v22n5a09.pdf

24. Suskind AM, Cawthon PM, Nakagawa S, Subak LL, Reinders I, Satterfield S, et al. Urinary incontinence in older women: the role of body composition and muscle strength: from de heath, aging, and body composition study. J Am Geriatr.Soc. [Internet].2017[citado $2018 \mathrm{Abr}$ 21]: 65(1):42-50.Disponivelem: https://www.ncbi.nlm.nih.gov/pmc/articles/PMC5258849/

25. Parque S, Yeoum S, Kim Y, KwonHJ. Self-management experiences o folder Korean womenwithurinaryincontinence: a descriptivequalitativestudyusingfocusgroups. J WoundOstomyContinenceNurs. [Internet]. 2017 [citado 2018 Abr 21]; 44(6)572-577. Disponivel em: https://www.ncbi.nlm.nih.gov/pubmed/29117084 